\title{
Counting Lattice Paths With Four Types of Steps
}

\author{
Maciej Dziemiańczuk
}

Received: 7 March 2013 / Revised: 13 July 2013 / Published online: 14 September 2013

(C) The Author(s) 2013. This article is published with open access at Springerlink.com

\begin{abstract}
The main purpose of this paper is to derive generating functions for the numbers of lattice paths running from $(0,0)$ to any $(n, k)$ in $\mathbb{Z} \times \mathbb{N}$ consisting of four types of steps: horizontal $H=(1,0)$, vertical $V=(0,1)$, diagonal $D=(1,1)$, and sloping $L=(-1,1)$. These paths generalize the well-known Delannoy paths which consist of steps $H, V$, and $D$. Several restrictions are considered. However, we mainly treat with those which will be needed to get the generating function for the numbers $R(n, k)$ of these lattice paths whose points lie in the integer rectangle $\left\{(x, y) \in \mathbb{N}^{2}: 0 \leq x \leq n, 0 \leq y \leq k\right\}$. Recurrence relation, generating functions and explicit formulas are given. We show that most of considered numbers define Riordan arrays.
\end{abstract}

Keywords Enumeration of lattice paths · Generating functions

Mathematics Subject Classification (2000) 05A15 - 05C30

\section{Introduction}

We follow the notation of Lehner [9] and Chen et al. [2]. A lattice path is a sequence of points in the integer lattice $\mathbb{Z}^{2}$. A pair of consecutive points is called a step of the path. Let $\sigma=\left(\sigma_{1}, \sigma_{2}\right)$ be a step. By writing, for instance, $\sigma$ is a step $H=(1,0)$ we mean that $\sigma_{2}-\sigma_{1}=H$.

Let us consider the family of lattice paths running from $(0,0)$ to $(n, k)$ and consisting of horizontal steps $H=(1,0)$ and vertical steps $V=(0,1)$. It is well known that the number of them is

M. Dziemiańczuk $(\bowtie)$

Institute of Informatics, University of Gdańsk, st. Wita Stwosza 57,

80-952 Gdańsk, Poland

e-mail: mdziemia@inf.ug.edu.pl 
Fig. 1 A Delannoy path from the origin to $(4,3)$

Fig. 2 A lattice path from $\mathbf{R}(4,3)$

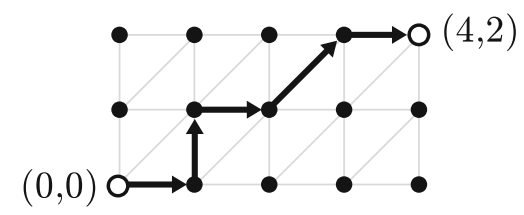

$(0,0)$

$(0,0)$

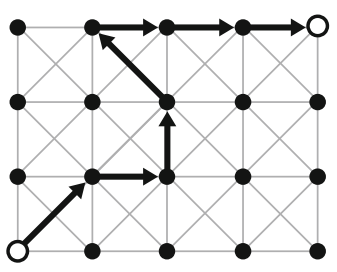

$(4,3)$

$$
\left(\begin{array}{c}
n+k \\
k
\end{array}\right)=\left[z^{k}\right] \frac{1}{(1-z)^{n+1}}
$$

Adding third kind of steps, diagonal ones $D=(1,1)$, we obtain Delannoy paths (see Fig. 1) and corresponding Delannoy numbers $D(n, k)$. That is, $D(n, k)$ is the number of lattice paths running from $(0,0)$ to $(n, k)$ consisting of horizontal $H$, vertical $V$ and diagonal steps $D=(1,1)$. See Comtet [3, p. 81] and Stanley [16, p. 185]. We refer the reader to Banderier and Schwer [1] and to the references given there. In this case we have

$$
D(n, k)=\sum_{i \geq 0}^{k}\left(\begin{array}{l}
n \\
i
\end{array}\right)\left(\begin{array}{c}
n+k-i \\
n
\end{array}\right)=\left[z^{k}\right] \frac{(1+z)^{n}}{(1-z)^{n+1}}
$$

Continue in this fashion we add fourth kind of steps, so-called sloping steps $L=$ $(-1,1)$, and we ask about the number of paths running from $(0,0)$ to $(n, k)$ in this generalized case. There are several variants which we may deal with. However, we mainly treat with those which will be needed to get the generating functions for the numbers $R(n, k)$ of these lattice paths from the origin to $(n, k)$ whose points lie entirely in the integer rectangle of lattice points $\{(i, j): 0 \leq i \leq n, 0 \leq j \leq k\}$ (see Fig. 2 for $n=4, k=3)$. In Sect. 6 we show that

$$
R(n, k)=\left[z^{k}\right] \frac{2^{n+2}(1+z)^{n} \sqrt{1-6 z-3 z^{2}}}{\left(1-z+\sqrt{1-6 z-3 z^{2}}\right)^{n+2}-\left(1-z-\sqrt{1-6 z-3 z^{2}}\right)^{n+2}} .
$$

However, simple exact formula for $R(n, k)$ is still unknown. Here we give the array $(R(n, k))$ for $0 \leq n \leq 6$ and $0 \leq k \leq 8$, 


$$
\left(\begin{array}{lllllllll}
1 & 1 & 1 & 1 & 1 & 1 & 1 & 1 & 1 \\
1 & 4 & 12 & 36 & 108 & 324 & 972 & 2916 & 8748 \\
1 & 7 & 33 & 143 & 609 & 2583 & 10945 & 46367 & 196417 \\
1 & 10 & 63 & 341 & 1748 & 8773 & 43653 & 216434 & 1071483 \\
1 & 13 & 102 & 656 & 3860 & 21756 & 119948 & 653612 & 3539052 \\
1 & 16 & 150 & 1115 & 7376 & 45801 & 274243 & 1606727 & 9288000 \\
1 & 19 & 207 & 1745 & 12809 & 86739 & 558967 & 3489601 & 21333553
\end{array}\right) .
$$

Before we present other results let us specify the notation. We write $\mathbb{N}$ for the set $\{0,1, \ldots\}$. Let $\mathcal{L}$ denote the set of integer lattice points $\{(i, j) \in \mathbb{Z} \times \mathbb{N}\}$. By the positive lattice $\mathcal{L}^{+}$and negative lattice $\mathcal{L}^{-}$we mean the sets $\left\{(i, j) \in \mathbb{N}^{2}\right\}$ and $\{(i, j) \in \mathbb{Z} \times \mathbb{N}$ : $i \leq 0\}$, respectively. Note that we include positive points of the $y$-axis in both cases. We also write $\mathcal{L}^{*}=\left\{(i, j) \in \mathbb{N}^{2}: i \geq j\right\}$ and $\mathcal{L}_{n}=\left\{(i, j) \in \mathbb{N}^{2}: 0 \leq i \leq n\right\}$.

Let $\mathcal{I}$ be a subset of $\mathcal{L}$. From now on and throughout the paper, by the lattice path $\pi$ of $\mathcal{I}$ we mean a sequence $\left(\pi_{1}, \pi_{2}, \ldots, \pi_{j}\right)$ of adjacent steps $\pi_{i}$ of four types: horizontal $(1,0)$, vertical $(0,1)$, diagonal $(1,1)$, and sloping $(-1,1)$, and whose set of points is a subset of $\mathcal{I}$. Let $(x, y)$ be a lattice point and $\pi$ a lattice path, we write $(x, y) \in \pi$ if $(x, y)$ is a point of $\pi$.

\subsection{List of Variants}

Let $X \in\{S, P, N, U, R\}$. By $X(n, k)$ we denote the number of paths running from $(0,0)$ to $(n, k) \in \mathbb{Z} \times \mathbb{N}$ with certain restrictions described in the following list. In bold print $\mathbf{X}$ we denote corresponding family of paths and by calligraphic letter $\mathcal{X}$ we denote certain generating function of $X$.

1. $S(n, k)$-paths of $\mathcal{L}$ (see Fig. 3a),

2. $P(n, k)$-paths of $\mathcal{L}^{+}$(see Fig. 3b),

3. $N(n, k)$-paths of $\mathcal{L}^{-}$(see Fig. 4a),

4. $U(n, k)$-paths of $\mathcal{L}^{*}$ (see Fig. $4 \mathrm{~b}$ ),

5. $R(n, k)$-paths of $\mathcal{L}_{n}$ (see Fig. 2).

These paths are connected with weighted free $(t, l)$-Motzkin paths [2]. A weighted free $(t, l)$-Motzkin path is a lattice path from $(0,0)$ to $(n, 0)$ consisting of horizontal steps $(1,0)$, down steps $(1,-1)$, and up steps $(1,1)$, and for which each of horizontal and down steps have been assigned a number from the sets $\{1,2, \ldots, t\}$ and $\{1,2, \ldots, l\}$, respectively. It turns out [5] that there is a bijection between $\mathbf{S}(0, n)$ and the family of weighted free $(3,3)$-Motzkin paths of length $n$.

\subsection{List of Generating Functions}

In the first part of the paper we show that

$$
\mathcal{S}(z)=\sum_{n \geq 0} S(0, n) z^{n}=\frac{1}{\sqrt{1-6 z-3 z^{2}}}
$$




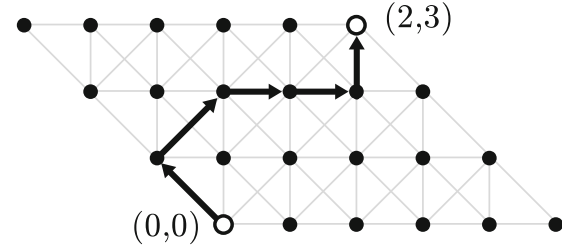

(a)

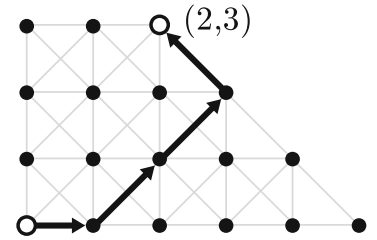

(b)

Fig. 3 Two lattice paths: a one from $\mathbf{S}(2,3)$ and $\mathbf{b}$ another from $\mathbf{P}(2,3)$

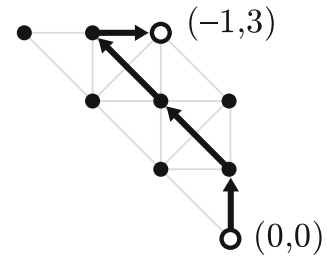

(a)

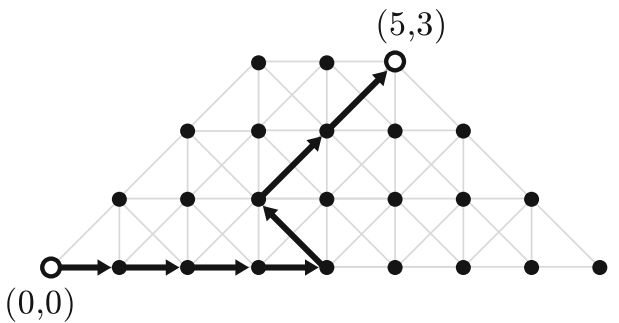

(b)

Fig. 4 Two lattice paths: $\mathbf{a}$ one from $\mathbf{N}(-1,3)$ and $\mathbf{b}$ another from $\mathbf{U}(5,3)$

$$
\begin{aligned}
& \widehat{\mathcal{S}}(z)=\sum_{n \geq 0} S(n, n) z^{n}=\left(\frac{3-2 z}{3}\left(2 \cos \left[\frac{2}{3} \arcsin \left[\frac{-36+7 z}{(-6+4 z) \sqrt{\frac{3-2 z}{z}}}\right]-1\right]\right)\right)^{-1}, \\
& \mathcal{P}(z)=\sum_{n \geq 0} P(0, n) z^{n}=\frac{1-z-\sqrt{1-6 z-3 z^{2}}}{2 z(z+1)}, \\
& \mathcal{U}(z)=\sum_{n \geq 0} U(n, n) z^{n}=-\frac{1}{3}-\frac{2}{3} \sqrt{\frac{3-2 z}{z}} \sin \left\{\frac{1}{3} \arcsin \left(\frac{(36-7 z) \sqrt{\frac{z}{3-2 z}}}{-6+4 z}\right)\right\} .
\end{aligned}
$$

In the next part we generalize the results listed above. For instance, for the lattice $\mathcal{L}$ we show that

$$
\begin{aligned}
\sum_{n \geq 0} \sum_{k \geq 0} S(n, k) z^{k} t^{n} & =\frac{\mathcal{S}(z)}{1-\mathcal{P}(z)(1+z) t}, \\
\sum_{n \geq 0} \sum_{k \geq 0} S(-n, n+k) z^{k} t^{n} & =\frac{\mathcal{S}(z)}{1-\mathcal{P}(z) t}, \\
\sum_{n \geq 0} \sum_{k \geq 0} S(n+k, k) z^{k} t^{n} & =\frac{\widehat{\mathcal{S}}(z)}{1-\mathcal{U}(z) t} .
\end{aligned}
$$


For the lattices $\mathcal{L}^{+}, \mathcal{L}^{-}$, and $\mathcal{L}^{*}$ we obtain

$$
\begin{aligned}
\sum_{n \geq 0} \sum_{k \geq 0} P(n, k) z^{k} t^{n} & =\frac{\mathcal{P}(z)}{1-\mathcal{P}(z)(1+z) t}, \\
\sum_{n \geq 0} \sum_{k \geq 0} N(-n, n+k) z^{k} t^{n} & =\frac{\mathcal{P}(z)}{1-\mathcal{P}(z) t}, \\
\sum_{n \geq 0} \sum_{k \geq 0} U(n+k, k) z^{k} t^{n} & =\frac{\mathcal{U}(z)}{1-\mathcal{U}(z) t} .
\end{aligned}
$$

In Sect. 7 we derive a generating function for the numbers $G(n)$ which count lattice paths from the origin to any point of the line $x=0$, and whose length is $n$. We get

$$
\mathcal{G}(z)=\sum_{k \geq 0} G(k) z^{k}=\frac{1-t-\sqrt{1-2 z-7 z^{2}}}{4 z^{2}} .
$$

In Sect. 8 we show that most of these numbers define certain Riordan arrays.

As for prerequisites, the reader is expected to be familiar with generating functions manipulation and extraction of coefficients. The standard work on these techniques is the book of Wilf [18]. These methods are called also as the method of coefficients, see Merlini et al. [11] for the compactly review of generating functions tools, connections between g.f., Riordan arrays and inversion formulas.

\section{The Base Case}

We denote by $\mathbf{S}(n, k)$ the family of lattice paths from $(0,0)$ to $(n, k)$ of the lattice $\mathcal{L}$, and by $S(n, k)$ the size of that family. An example of a path from $\mathbf{S}(2,3)$ is given in Fig. 3a. The last step of any path from $\mathbf{S}(n, k)$ is one of horizontal, vertical, diagonal, or sloping. It implies that the numbers $S(n, k)$ satisfy the following four-term recurrence relation, $S(-k, k)=1$ for $k \geq 0$, and

$$
S(n, k)=S(n-1, k)+S(n, k-1)+S(n-1, k-1)+S(n+1, k-1)
$$

for $k \geq 0$ and $n \geq-k$. Using standard methods (see e.g. Wilf [18]) we derive its ordinary generating functions from the recurrence relation, i.e.,

$$
\begin{aligned}
\sum_{k \geq 0} \sum_{n \geq 0} S(n-k, k) x^{n} y^{k} & =\frac{1}{1-x-y-x y-x^{2} y} \\
\sum_{n \geq 0} S(n-k, k) x^{n} & =\frac{\left(1+x+x^{2}\right)^{k}}{(1-x)^{k+1}}
\end{aligned}
$$




$$
\mathcal{S}_{\langle n\rangle}(y)=\sum_{k \geq 0} S(n-k, k) y^{k}=\frac{\alpha_{+}(y)^{n+1}-\alpha_{-}(y)^{n+1}}{\sqrt{1+6 y-3 y^{2}}},
$$

where $\alpha_{ \pm}(y)=\left(1+y \pm \sqrt{1+6 y-3 y^{2}}\right) /(2(1-y))$.

Proposition 1 For every $k \geq 0$ and $n \geq-k$, we have

$$
S(n, k)=\sum_{i=0}^{n+k} \sum_{j=0}^{i}\left(\begin{array}{l}
k \\
j
\end{array}\right)\left(\begin{array}{c}
j \\
i-j
\end{array}\right)\left(\begin{array}{c}
2 k+n-i \\
k
\end{array}\right) .
$$

Proof The generating function (2) is the product of $\left(1+x+x^{2}\right)^{k}$ and $1 /(1-x)^{k+1}$. Thus $S(n-k, k)=\sum_{i=0}^{n}\left[x^{i}\right]\left(1+x+x^{2}\right)^{k}\left[x^{n-i}\right] 1 /(1-x)^{k+1}$. The coefficient of $x^{n}$ in the series expansion of $1 /(1-x)^{k+1}$ is $\left(\begin{array}{c}n+k \\ n\end{array}\right)$. To find the series expansion of $\left(1+x+x^{2}\right)^{k}$ we use the binomial theorem for $(1+x(1+x))^{k}$. In general, we have

$$
\left[x^{n}\right]\left(\alpha+\beta x+\gamma x^{2}\right)^{k}=\sum_{j=0}^{n}\left(\begin{array}{l}
k \\
j
\end{array}\right)\left(\begin{array}{c}
j \\
n-j
\end{array}\right) \alpha^{k-j} \beta^{2 j-n} \gamma^{n-j} .
$$

Relabelling $n \mapsto n+k$ we obtain the formula for $S(n, k)$.

Remark Another approach to finding the coefficient of the series expansions of the functions similar to $\left(1+u+u^{2}\right)^{n}$ follows to the so-called "composita" introduced and developed by Kruchinin [8].

\section{Counting Paths that Lie in Triangles}

Let us observe that paths from $\mathbf{S}(n, k)$ contain lattice points of a discrete parallelogram of sizes $n \times k$ (see Fig. 3a). In this section we consider paths that lie in certain discrete triangles of lattice points.

\subsection{The Right Triangle}

We denote by $\mathbf{P}(n, k)$ the family of lattice paths from $(0,0)$ to $(n, k)$ whose points lie in the positive lattice $\mathcal{L}^{+}$(see Fig. 5b). We write $P(n, k)=|\mathbf{P}(n, k)|$ and $P(k)=P(0, k)$.

Proposition $2 P(0)=1$, and for every $n \geq 1$, we have

$$
P(n)=P(n-1)+\sum_{i=0}^{n-1} P(i) P(n-1-i)+\sum_{i=0}^{n-2} P(i) P(n-2-i) .
$$

Proof Observe that we can separate $\mathbf{P}(0, n)$ into two disjoint classes $A$ and $B$, such that $A$ and $B$ contain these paths whose the last steps are vertical $((0, n-1),(0, n))$ and sloping $((1, n-1),(0, n))$, respectively. Therefore, $|A|=|\mathbf{P}(0, n-1)|$ and $|B|=|\mathbf{P}(1, n-1)|$. The size of $A$ is $P(n-1)$. 


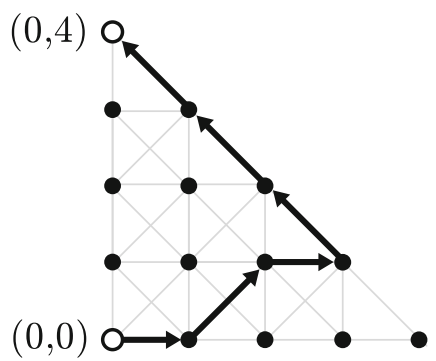

(a)

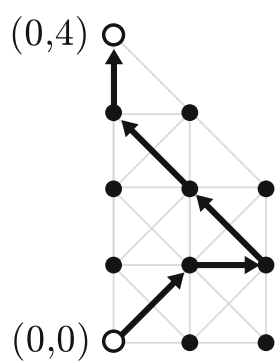

(b)

Fig. 5 a A path from $\mathbf{P}(0,4)$ and $\mathbf{b}$ another one from $\mathbf{P}_{2}(0,4)$

To calculate the size of $B$, observe that every path $\pi$ from $B$ must have a step $h_{i}=((0, i),(1, i))$ or $d_{i}=((0, i),(1, i+1))$, where $i=\max \left\{j: h_{j} \in \pi\right.$ or $\left.d_{j} \in \pi\right\}$. If $\pi$ has $h_{i}$, then $\pi$ can be uniquely decomposed into $\pi_{1} h_{i} \pi_{2}$, where $\pi_{1}$ and $\pi_{2}$ are paths from $\mathbf{P}(i)$ and $\mathbf{P}(n-1-i)$, respectively. On the other hand, if $\pi$ has $d_{i}$, then it can be uniquely decomposed into $\pi_{1} d_{i} \pi_{2}$, where $\pi_{1}$ and $\pi_{2}$ are paths from $\mathbf{P}(i)$ and $\mathbf{P}(n-2-i)$, respectively. It determines the partition of $B$ into classes $H_{0}, \ldots, H_{n-1}$ and $D_{0}, \ldots, D_{n-2}$, where the sizes of $H_{i}$ and $D_{i}$ are $P(i) P(n-1-i)$ and $P(i) P(n-2-i)$, respectively.

Let $\mathcal{P}(z)=\sum_{k \geq 0} P(k) z^{k}$, then

$$
\mathcal{P}(z)=\frac{1-z-\sqrt{1-6 z-3 z^{2}}}{2 z(z+1)} .
$$

The explicit form of $\mathcal{P}(z)$ was given by Brian Drake [4, eq. 11] by using theory of sequences that count lattice paths by area. However, it can be inferred from Proposition 2. Indeed, the recurrence (5) implies

$$
\mathcal{P}(z)=z \mathcal{P}(z)+z \mathcal{P}(z)^{2}+z^{2} \mathcal{P}(z)^{2}+1
$$

Thus $\mathcal{P}(z)$ is that root of the functional equation $\left(z+z^{2}\right) \mathcal{P}(z)^{2}+(z-1) \mathcal{P}(z)+1=0$, which satisfies an initial condition $\left[z^{0}\right] \mathcal{P}(z)=P(0)=1$. This fact was indicated to the author by Vladimir Kruchinin. Simplifying (7) we obtain $\mathcal{P}(z)=1 /(1-z-(z+$ $\left.\left.z^{2}\right) \mathcal{P}(z)\right)$ which can be written as a continued fraction

$$
\mathcal{P}(z)=\frac{1}{1-z-\frac{z+z^{2}}{1-z-\frac{z+z^{2}}{1-z-\frac{z+z^{2}}{1-z-\ldots}}}} .
$$

We shall use this representation of $\mathcal{P}(z)$ in the next section. 
Theorem 1 For all $n \geq 0$ and $d \geq 1$, we have

$$
\left[z^{n}\right] \mathcal{P}(z)^{d}=\frac{d}{n+d} \sum_{k=0}^{n} \sum_{j=0}^{k}\left(\begin{array}{c}
n+d \\
j
\end{array}\right)\left(\begin{array}{c}
j \\
k-j
\end{array}\right)\left(\begin{array}{c}
2 n+d-k-1 \\
n-1+d
\end{array}\right)
$$

Proof Multiplying both sides of (7) by $z$ and substituting $u=z \mathcal{P}(z)$ we see that $\left[z^{n}\right] \mathcal{P}(z)^{d}=\left[z^{n+d}\right] u(z)^{d}$. Let us rewrite $(7)$ as $u=z\left(1+u+u^{2}\right) /(1-u)$. Observe that $u=z \phi(u)$, where $\phi(u)=\left(1+u+u^{2}\right) /(1-u)$ and $\phi(0)=1$. From the Lagrange Inversion Formula (see e.g. Merlini et al. [10, Eq. 2.1]) we obtain

$$
\left[z^{n}\right]\left\{u(z)^{d}\right\}=\frac{d}{n}\left[u^{n-d}\right]\left\{\left(\frac{1+u+u^{2}}{1-u}\right)^{n}\right\} .
$$

We deal with the series expansion of $\left(1+u+u^{2}\right)^{n}$ in much the same way as in Proposition 1.

Corollary 1 For all $n \geq 0$ and $d \geq 1$, we have

$$
\left[z^{n}\right] \mathcal{P}(z)^{d}=\frac{d}{d+n}(S(-d, d+n)-S(-d-1, d+n)) .
$$

Proof Observe that the function $\left(1+u+u^{2}\right)^{n} /(1-u)^{n}$ in (9) is the generating function (2) multiplied by $(1-u)$. Simple modification of (9) gives the formula.

Corollary 2 For $n \geq 0$ we have

$$
\begin{aligned}
& P(n)=\frac{1}{n+1}(S(-2, n)+S(-1, n)+S(0, n)), \\
& P(n)=\frac{1}{n+1} \sum_{k=0}^{n} \sum_{j=0}^{k}\left(\begin{array}{c}
n+1 \\
j
\end{array}\right)\left(\begin{array}{c}
j \\
k-j
\end{array}\right)\left(\begin{array}{c}
2 n-k \\
n
\end{array}\right) .
\end{aligned}
$$

Proof The first equation follows from Corollary 1 for $d=1$ with the recurrence relation for $S(-1, n+1)$, i.e., $S(-1, n+1)=S(-2, n+1)+S(-2, n)+S(-1, n)+$ $S(0, n)$. The second equation follows from Theorem 1 for $d=1$.

Problem 1 Find a combinatorial proof of (10).

Let us compare the generating function $\mathcal{P}(z)$ with $\alpha_{ \pm}(z)$ from the previous section and note that $\alpha_{-}(z)=-z \mathcal{P}(-z)$. Thus $\left[z^{n}\right] \alpha_{-}(z)=(-1)^{n} P(n-1)$ and $\left[z^{n}\right] \alpha_{+}(z)=$ $(-1)^{n+1} P(n-1)+2$.

Remark The sequence

$$
(P(n))_{n \geq 0}=(1,2,7,29,133,650,3319,17498,94525,520508,2910895, \ldots)
$$

is denoted by A064641 in OEIS [13]. 


\subsection{Generalized Right Triangle Paths}

Let $\mathbf{P}_{n}(p, k)$ denote the family of lattice paths running from $(0,0)$ to $(p, k)$ that lie in the lattice $\mathcal{L}_{n}$. We write $T_{n}(k)=\left|\mathbf{P}_{n}(0, k)\right|$. We have $T_{n}(k)=P(k)$ for $n \geq k$. An example of a path from $\mathbf{P}_{2}(0,4)$ is given in Fig. $5 \mathrm{~b}$.

Proposition 3 We have $T_{n}(0)=1$ for $n \geq 0, T_{0}(k)=1$ for $k \geq 0$, and

$$
T_{n}(k)=T_{n}(k-1)+\sum_{i=0}^{k-1} T_{n}(i) T_{n-1}(k-1-i)+\sum_{i=0}^{k-2} T_{n}(i) T_{n-1}(k-2-i) .
$$

Proof We prove it in much the same way as Proposition 2.

Theorem 2 For $n \geq 0$ we have

$$
\mathcal{T}_{n}(z)=\sum_{k \geq 0} T_{n}(k) z^{k}=2 \frac{\beta_{+}(z)^{n+1}-\beta_{-}(z)^{n+1}}{\beta_{+}(z)^{n+2}-\beta_{-}(z)^{n+2}},
$$

where $\beta_{ \pm}(z)=\left(1-z \pm \sqrt{1-6 z-3 z^{2}}\right)$.

Proof From (12) it follows that $\mathcal{T}_{n}(z)=1 /\left(1-z-\mathcal{T}_{n-1}(z)\left(z+z^{2}\right)\right)$ with $\mathcal{T}_{0}(z)=$ $1 /(1-z)$. Let us consider $\mathcal{T}_{n}(z)$ as the $n$th convergent of the continued fraction (8), and define a sequence of partial numerators $A_{n}$ as follows, $A_{0}=0, A_{1}=1$ and $A_{n}=(1-z) A_{n-1}-z(z+1) A_{n-2}$ for $n \geq 0$. Simple verification shows that $\mathcal{T}_{n}(z)=A_{n+1} / A_{n+2}$. Solving the recurrence we find that

$$
A_{n}=\frac{\left(1-z+\sqrt{1-6 z-3 z^{2}}\right)^{n}-\left(1-z-\sqrt{1-6 z-3 z^{2}}\right)^{n}}{2^{n} \sqrt{1-6 z-3 z^{2}}},
$$

and the formula follows.

Let us give some first four examples of $\mathcal{T}_{n}(z)$,

- $\mathcal{T}_{0}(z)=1 /(1-z)$,

- $\mathcal{T}_{1}(z)=(1-z) /(1-3 z)$,

- $\mathcal{T}_{2}(z)=(1-3 z) /\left(1-5 z+3 z^{2}+z^{3}\right)$,

- $\mathcal{T}_{3}(z)=\left(1-5 z+3 z^{2}+z^{3}\right) /\left(1-7 z+10 z^{2}+z^{3}-z^{4}\right)$.

Problem 2 Find an exact formula for $T_{n}(k)$.

\subsection{The Isosceles Triangle}

We denote by $\mathbf{U}(n, k)$ the family of paths from $(0,0)$ to $(n, k)$ that lie in $\mathcal{L}^{*}$, and we write $U(n, k)=|\mathbf{U}(n, k)|$. An example of a path from $\mathbf{U}(5,3)$ is given in Fig. 4b. We set $U(n)=U(n, n)$. 
Proposition $4 U(0)=1$, and for $n \geq 1$ we have

$$
\begin{aligned}
U(n)=U(n-1) & +\sum_{i=0}^{n-1} U(i) U(n-1-i)+ \\
& +\sum_{i=0}^{n-1} \sum_{j=0}^{n-1-i} U(i) U(j) U(n-i-j-1) .
\end{aligned}
$$

Proof We partition the set $\mathbf{U}(n, n)$ into three classes $A, B$, and $C$, where $|A|=$ $|\mathbf{U}(n-1, n-1)|,|B|=|\mathbf{U}(n, n-1)|$ and $|C|=|\mathbf{U}(n+1, n-1)|$. The size of $A$ is $U(n-1)$. To calculate the size of $B$, observe that every path $\pi$ from $B$ must have a horizontal step $h_{i}=((i, i),(i+1, i))$, and suppose that $i=\max \left\{j: h_{j} \in \pi\right\}$. Thus $\pi$ can be uniquely decomposed as $\pi_{1} h_{i} \pi_{2}$, where $\pi_{1}$ and $\pi_{2}$ are paths from $\mathbf{U}(i)$ and $\mathbf{U}(n-1-i)$, respectively.

The class $C$ can be handled in much the same way as $B$. Except that now every path from $C$ must have two horizontal steps $h_{i}^{\prime}=((i, i),(i+1, i))$ and $h_{j}^{\prime \prime}=((j+$ $1, j),(j+2, j))$, where $j \geq i$.

Theorem $3 \operatorname{Let} \mathcal{U}(z)=\sum_{n \geq 0} U(n) z^{n}$, then

$$
\mathcal{U}(z)=-\frac{1}{3}-\frac{2}{3} \sqrt{\frac{3-2 z}{z}} \sin \left\{\frac{1}{3} \arcsin \left(\frac{(36-7 z) \sqrt{\frac{z}{3-2 z}}}{-6+4 z}\right)\right\}
$$

Proof From Proposition 4 we see that $\mathcal{U}(z)$ is that root of the following cubic equation

$$
1+(z-1) \mathcal{U}(z)+z \mathcal{U}(z)^{2}+z \mathcal{U}(z)^{3}=0
$$

which satisfies $\mathcal{U}(0)=U(0)=1$. We reduce (16) to the canonical form $u^{3}+p u+q=$ 0 , where $\mathcal{U}(z)=u-1 / 3, p=2 / 3-1 / z, q=-7 / 27+4 /(3 z)$. The $k$ th root of the equation, for $k=0,1,2$, is

$$
u_{k}=\frac{2}{3} \sqrt{\frac{3-2 z}{z}} \cos \left\{\frac{1}{3}\left[\arccos \left(\frac{(36-7 z) \sqrt{\frac{z}{3-2 z}}}{-6+4 z}\right)-2 k \pi\right]\right\}
$$

The required root is $u_{1}$ due to $\lim _{z->0}\left(u_{1}-1 / 4\right)=1$. What is left is to substitute back with $\mathcal{U}(z)=u_{1}-1 / 3$. Using the standard properties of $\sin$ and cos we obtain the formula.

Theorem 4 For $d \geq 0$ we have $\left[z^{0}\right] \mathcal{U}(z)^{d}=1$, and for $n \geq 1$ we have

$$
\left[z^{n}\right] \mathcal{U}(z)^{d}=\sum_{p=0}^{d} \sum_{k=0}^{n-p} \sum_{i=0}^{n-p-k} \frac{p}{n}\left(\begin{array}{l}
d \\
p
\end{array}\right)\left(\begin{array}{l}
n \\
k
\end{array}\right)\left(\begin{array}{l}
n \\
i
\end{array}\right)\left(\begin{array}{c}
i \\
n-p-k-i
\end{array}\right) 3^{i+p+k}
$$


Proof The function $\mathcal{U}(z)$ satisfies the functional Eq. (16). Letting $u=u(z)=\mathcal{U}(z)-1$ we find that $u=z \phi(u)$, where $\phi(u)=(u+1)\left(3+3 u+u^{2}\right)$. By the Lagrange Inversion Formula,

$$
\begin{aligned}
{\left[z^{n}\right] u(z)^{d} } & =\frac{d}{n}\left[u^{n-d}\right]\left\{(u+1)^{n}\left(3+3 u+u^{2}\right)^{n}\right\} \\
& =\frac{d}{n} \sum_{k=0}^{n-d}\left(\begin{array}{l}
n \\
k
\end{array}\right) \gamma(n, n-d-k),
\end{aligned}
$$

where $\gamma(n, j)=\left[u^{j}\right]\left(3+3 u+u^{2}\right)^{n}$. From (4) we see that $\gamma(n, j)$ is equal to $\sum_{i=0}^{j}\left(\begin{array}{c}n \\ i\end{array}\right)\left(\begin{array}{c}i \\ j-i\end{array}\right) 3^{n+i-j}$. We have now a formula for the coefficients of the series expansion of $u(z)^{d}$. Let us substitute back $\mathcal{U}(z)=u+1$ to obtain the desired formula, i.e.,

$\left[z^{n}\right] \mathcal{U}^{d}(z)=\left[z^{n}\right](u+1)^{d}=\sum_{p=0}^{d}\left(\begin{array}{l}d \\ p\end{array}\right)\left[z^{n}\right] u^{p}=\sum_{p=0}^{d} \sum_{k=0}^{n-p} \frac{p}{n}\left(\begin{array}{l}d \\ p\end{array}\right)\left(\begin{array}{l}n \\ k\end{array}\right) \gamma(n, n-p-k)$

Corollary $3 U(0)=1$, and for $n \geq 1$ we have

$$
U(n)=\frac{3}{n} \sum_{k=0}^{n-1} \sum_{i=0}^{n-1-k}\left(\begin{array}{l}
n \\
k
\end{array}\right)\left(\begin{array}{l}
n \\
i
\end{array}\right)\left(\begin{array}{c}
i \\
n-1-k-i
\end{array}\right) 3^{i+k}
$$

Proof We apply Theorem 4 for $d=1$.

Remark The sequence

$$
(U(n))_{n \geq 0}=(1,3,18,144,1323,13176,138348,1507977,16900650, \ldots)
$$

is denoted by A107708 in OEIS [13].

\section{Central Numbers of the Base Case}

In this section we obtain generating function for the numbers $S(0, n)$ and $S(n, n)$.

Theorem 5 The following statements are true and equivalent.

(a) (recurrence relation) $S(0,0)=1$, and for $n \geq 1$ we have

$$
S(0, n)=\sum_{k=1}^{n}\left(2 P(k-2)+2 P(k-1)+\delta_{k, 1}\right) S(0, n-k)
$$

where $\delta_{k, 1}=1$ for $k=1$, and zero otherwise. 
(b) (generating function)

$$
\mathcal{S}(z)=\sum_{n \geq 0} S(0, n) z^{n}=\frac{1}{\sqrt{1-6 z-3 z^{2}}}
$$

(c) (exact formula) For $n \geq 0$ we have

$$
S(0, n)=\frac{1}{2^{n}} \sum_{k=0}^{n}\left(\begin{array}{c}
2 k \\
k
\end{array}\right)\left(\begin{array}{c}
k \\
n-k
\end{array}\right) 3^{k}
$$

Proof (a) Let us partition the family $\mathbf{S}(0, n)$ into $n$ classes $A_{1}, \ldots, A_{n}$ such that $A_{k}$ contains these paths $\pi$ of $\mathbf{S}(0, n)$ for which we have $k=\min \{j \geq 1$ : $(0, j) \in \pi\}$. Take $\pi \in A_{k}$. We have three cases: (i) the first step of $\pi$ is vertical $((0,0),(0,1))$ if $k=1$, or (ii) the path in $\pi$ between $(0,0)$ and $(0, k)$ lies in the positive lattice $\mathcal{L}^{+}$, or (iii) the path in $\pi$ between $(0,0)$ and $(0, k)$ lies in the negative lattice $\mathcal{L}^{-}$. In each case, $\pi$ do not contain any of points $(0, j)$ for $0<$ $j<k$. In the first case (i) the number of such paths is $S(0, n-1)$. In the second case (ii) we see that $\pi$ contains a sloping step $\sigma=((1, k-1),(0, k))$ and begins with either horizontal $h=((0,0),(1,0))$ or a diagonal one $d=((0,0),(1,1))$. The remaining steps between the first step of $\pi$ and $\sigma$ form a path of $\mathbf{P}(0, k-1)$ or $\mathbf{P}(0, k-2)$, respectively. On the other hand, the remaining steps between $(0, k)$ and $(0, n)$ form a path of $\mathbf{S}(0, n-k)$. Thus the number of these paths is $(P(k-1)+P(k-2)) S(0, n-k)$. The third case (iii) is symmetrical to (ii).

(b) Let $f(z)=\sum_{k \geq 1}\left(2 P(k-2)+2 P(k-1)+\delta_{k, 1}\right) z^{k}$. Then by (6), we have $f(z)=2 z^{2} \mathcal{P}(z)+2 z \mathcal{P}(z)+z=1-\sqrt{1-6 z-3 z^{2}}$. On the other hand, from (17) it follows that $\mathcal{S}(z)=1 /(1-f(z))$. It is defined correctly due to $f(0)=0$.

(c) It follows from the generalized binomial theorem applied to (18), i.e.,

$$
\left[z^{n}\right](1-z(6+3 z))^{-1 / 2}=\sum_{k=0}^{n}\left(\begin{array}{c}
-1 / 2 \\
k
\end{array}\right)\left(\begin{array}{c}
k \\
n-k
\end{array}\right)(-1)^{k} 6^{2 k-n} 3^{n-k}
$$

where

$$
\left(\begin{array}{c}
-1 / 2 \\
k
\end{array}\right)=(-1)^{k} \frac{1 \cdot 3 \cdot 5 \cdots(2 k-1)}{2^{k} k !} \frac{2 \cdot 4 \cdot 6 \cdots 2 k}{2^{k} k !}=(-1)^{k} \frac{1}{2^{2 k}}\left(\begin{array}{c}
2 k \\
k
\end{array}\right) .
$$

Due to the square root in (18) the author of [5] uses Darboux's lemma to get the asymptotic behaviour of the numbers $S(0, n)$, see e.g. the book of Flajolet and Sedgewick [6]. Namely, for $m, n \geq 0$ we have

$$
z_{1}^{n} S(0, n)=\frac{\sqrt{2+\sqrt{3}}}{2} \sum_{j=0}^{m}\left(\begin{array}{c}
j-\frac{1}{2} \\
j
\end{array}\right)\left(\begin{array}{c}
n-\frac{1}{2}-j \\
n
\end{array}\right)\left(\frac{2-\sqrt{3}}{4}\right)^{j}+O\left(n^{-m-3 / 2}\right)
$$


where $z_{1}=(-3+2 \sqrt{3}) / 3$. For $m=2$ we obtain

$$
\begin{aligned}
S(0, n) & \approx \frac{\sqrt{2+\sqrt{3}}}{2} \frac{(3+2 \sqrt{3})^{n}}{\sqrt{n \pi}}\left(1+\frac{1-\sqrt{3}}{8 n}+\frac{34-18 \sqrt{3}}{128 n^{2}}+\cdots\right) \\
& \approx 0.545 \frac{6.4641^{n}}{\sqrt{n}}\left(1-\frac{0.09}{n}+\frac{0.022}{n^{2}}+\cdots\right) .
\end{aligned}
$$

Remark The sequence

$$
(S(0, k))_{k \geq 0}=(1,3,15,81,459,2673,15849,95175,576963,3523257, \ldots)
$$

is denoted by A122868 in OEIS [13]. Let us observe that

$$
\frac{d}{d z} z \mathcal{P}(z)=\mathcal{S}(z) \mathcal{P}(z)(1-z \mathcal{P}(z))
$$

which gives another proof of (10).

Let

$$
\widehat{\mathcal{S}}(z)=\sum_{n \geq 0} S(n, n) z^{n}
$$

Theorem 6 The following statements are true and equivalent.

(a) (recurrence relation) $S(0,0)=1$, and for $n \geq 1$ we have

$$
\begin{aligned}
S(n, n)= & \sum_{k=1}^{n}\left(2(U(k-1)+U(k, k-1))+\delta_{k}\right. \\
& \left.+\sum_{i=0}^{k-1} U(i) U(k-1-i)\right) S(n-k, n-k)
\end{aligned}
$$

where $\delta_{k}=1$ for $k=1$, and zero otherwise.

(b) (generating function)

$$
\widehat{\mathcal{S}}(z)=\left(\frac{3-2 z}{3}\left(2 \cos \left[\frac{2}{3} \arcsin \left[\frac{-36+7 z}{(-6+4 z) \sqrt{\frac{3-2 z}{z}}}\right]-1\right]\right)\right)^{-1}
$$

Proof (a) It may be handled in much the same way as the proof of Theorem 5. Instead of using the numbers $P(n)$ we apply here the numbers $U(n)$. Further, in this case we need to observe that a subpath between the origin $(0,0)$ and $(k, k)$ may contain a sloping step $((i+1, i),(i, i+1))$ for $i=0,1, \ldots, k-1$, which gives additional sum over $i$ in (20). (b) From (20) it follows that $\widehat{\mathcal{S}}(z)=(1-g(z))^{-1}$, where 


$$
g(z)=\sum_{k \geq 1}\left(2(U(k-1)+U(k, k-1))+\delta_{k, 1}+\sum_{i=0}^{k-1} U(i) U(k-1-i)\right) z^{k}
$$

Combining Theorem 4 with Proposition 4 we obtain $g(z)=z \mathcal{U}(z)(2+3 \mathcal{U}(z))+z$. The function $(1-g(z))^{-1}$ is defined correctly due to $g(0)=0$. Straightforward simplifying gives the desired formula.

The sequence $(S(n, n))_{n \geq 0}$ begins with

$$
(S(n, n))_{n \geq 0}=(1,6,60,675,7992,97416,1209951,15227190, \ldots) .
$$

\section{General Cases}

In previous section we inferred the generating functions for the numbers $P(n), U(n)$, $S(0, n)$, and $S(n, n)$. Here we generalize these results to the numbers $P(n, k), U(n, k)$, $S(n, k)$, and $N(n, k)$.

\subsection{The Positive Lattice $\mathcal{L}^{+}$}

Here we give the array $(P(i, j))_{i, j}$ for $0 \leq i \leq 4$ and $0 \leq j \leq 7$,

$$
\left(\begin{array}{llllllll}
1 & 2 & 7 & 29 & 133 & 650 & 3319 & 17498 \\
1 & 5 & 22 & 104 & 517 & 2669 & 14179 & 77027 \\
1 & 8 & 46 & 251 & 1369 & 7541 & 42031 & 236933 \\
1 & 11 & 79 & 497 & 2986 & 17642 & 103696 & 609428 \\
1 & 14 & 121 & 869 & 5746 & 36482 & 226768 & 1393637
\end{array}\right)
$$

Proposition $5 P(0, k)=P(k)$ for $k \geq 0$, and for $n \geq 1$ we have

$$
\begin{aligned}
& P(n, k)=\sum_{i=0}^{k} P(i) P(n-1, k-i)+\sum_{i=0}^{k-1} P(i) P(n-1, k-1-i), \\
& P(n, k)=S(n, k)-\sum_{i=0}^{k-1} P(i) S(n+1, k-1-i) .
\end{aligned}
$$

Proof (a) If $n=0$, then $P(n, k)=P(k)$. Let $n \geq 1$. Observe that every path $\pi$ from $\mathbf{P}(n, k)$ can be uniquely decomposed as either $\pi_{1} h_{i} \pi_{2}$ or $\pi_{1} h_{i} \pi_{3}$, where $h_{i}$ and $d_{i}$ are steps $((0, i),(1, i))$ and $((0, i),(1, i+1))$, respectively, and $\pi_{1}, \pi_{2}$, and $\pi_{3}$ are paths from $\mathbf{P}(i), \mathbf{P}(n-1, k-i)$, and $\mathbf{P}(n-1, k-1-i)$, respectively. Note that we take the maximal such number $i$, i.e., $i=\max \left\{j: h_{i} \in \pi\right.$ or $\left.d_{i} \in \pi\right\}$. See Proposition 2 for more details.

(b) Observe that for all $n, k \geq 0$ the family of paths $\mathbf{P}(n, k)$ is the set $\mathbf{S}(n, k) \backslash A$, where $A$ is the set of these paths from $\mathbf{S}(n, k)$ which contain points from the set of lattice points $\{(i, j): i<0, j>0\}$. To calculate the size of $A$, we observe 
that every path $\pi$ from $A$ can be uniquely decomposed as $\pi_{1} s_{i} \pi_{2}$, where $\pi_{1}, \pi_{2}$ are paths from $\mathbf{P}(0, i)$ and $\mathbf{S}(n+1, k-1-i)$, respectively, and $s_{i}$ is a sloping step $((0, i),(-1, i+1))$, for $i \in\{0,1, \ldots, k-1\}$. It determines the partition of $A$ into classes $A_{0}, \ldots, A_{k-1}$, where $\left|A_{i}\right|=P(i) S(n+1, k-1-i)$.

Theorem 7 For $n \geq 0$ we have

$$
\begin{gathered}
\mathcal{P}(x, z)=\sum_{n, k \geq 0} P(n, k) x^{n} z^{k}=\frac{\mathcal{P}(z)}{1-(1+z) \mathcal{P}(z) x}, \\
\mathcal{P}_{n}(z)=\sum_{k \geq 0} P(n, k) z^{k}=\mathcal{P}(z)^{n+1}(1+z)^{n} .
\end{gathered}
$$

Proof Multiplying both sides of (22a) by $z^{k}$ and summing over $k \geq 0$ we obtain the functional equation $\mathcal{P}_{n}(z)=\mathcal{P}(z) \mathcal{P}_{n-1}(z)+z \mathcal{P}(z) \mathcal{P}_{n-1}(z)$ with $\mathcal{P}_{0}(z)=\mathcal{P}(z)$. Solving it we derive (23b). Next, from (23b) we obtain (23a).

Combining (6) with (23a) we obtain

$$
\mathcal{P}(x, y)=\sum_{n, k \geq 0} P(n, k) x^{n} y^{k}=\frac{2}{1-y+\sqrt{1-6 y-3 y^{2}}-2(1+y) x} .
$$

Corollary 4 For all $n, k \geq 0$ we have

$$
P(n, k)=\sum_{r=0}^{k} \sum_{s=0}^{r} \sum_{j=0}^{s} \frac{n+1}{r+n+1}\left(\begin{array}{c}
n \\
k-r
\end{array}\right)\left(\begin{array}{c}
2 r+n-s \\
r+n
\end{array}\right)\left(\begin{array}{c}
r+n+1 \\
j
\end{array}\right)\left(\begin{array}{c}
j \\
s-j
\end{array}\right) .
$$

Proof Applying Theorem 1 to (23b) gives the coefficients of the series expansion of $\mathcal{P}(y)^{n+1}$. Using the product rule of the series $\mathcal{P}(y)^{n+1}$ and $(1+y)^{n}$ we obtain the formula.

Corollary 5 We have

$$
\mathcal{P}(z)=\mathcal{S}(z)\left(1-z \mathcal{P}(z)^{2}(1+z)\right)
$$

Proof Applying (22b) for $n=0$ we obtain a recurrence relation for the numbers $P(k)$. The recurrence implies the desired functional equation.

Using residue theorem to $\mathcal{P}(x / z, z)$ we may obtain the following generating function.

Conjecture 1 We have

$$
\sum_{n \geq 0} P(n, n) x^{n}=\left.\frac{z-z^{2}-2 x(1+z)-z \sqrt{1-6 z-3 z^{2}}}{2(1+z)\left(-x+x^{2}+2 x z+3 z^{2}\right)}\right|_{z \rightarrow x \mathcal{U}(x)}
$$


Remark The sequence $P(n, n)$ begins with

$$
(P(n, n))_{n \geq 0}=(1,5,46,497,5746,68948,846889,10570001, \ldots) .
$$

\subsection{The Negative Lattice $\mathcal{L}^{-}$}

We denote by $\mathbf{N}(-n, n+k)$ the family of paths from $(0,0)$ to $(-n, n+k)$ that lie in the negative lattice $\mathcal{L}^{-}$. The size of that family is denoted by $N(-n, n+k)$. Here we give the $(N(-i, i+j))_{i, j}$ for $0 \leq i \leq 4$ and $0 \leq j \leq 7$,

$$
\left(\begin{array}{llllllll}
1 & 2 & 7 & 29 & 133 & 650 & 3319 & 17498 \\
1 & 4 & 18 & 86 & 431 & 2238 & 11941 & 65086 \\
1 & 6 & 33 & 179 & 978 & 5406 & 30241 & 171045 \\
1 & 8 & 52 & 316 & 1874 & 11020 & 64698 & 380400 \\
1 & 10 & 75 & 505 & 3235 & 20202 & 124455 & 761160
\end{array}\right) .
$$

Proposition 6 For $k \geq 0$ we have $N(0, k)=P(k)$, and for $n \geq 1$ we have

$$
N(-n, n+k)=\sum_{i=0}^{k} P(i) N(-n+1, n-1+k-i) .
$$

Proof We have $N(0, k)=P(k)$ for $k \geq 0$. Let $n \geq 1$, and observe that every path $\pi$ from $\mathbf{N}(-n, n+k)$ must have a sloping step $s_{i}=((0, i),(-1, i+1))$, where $i=\max \left\{j: s_{j} \in \pi\right\}$. Thus $\pi$ can be uniquely decomposed as $\pi_{1} s_{i} \pi_{2}$, where $\pi_{1}$ and $\pi_{2}$ are paths from $\mathbf{P}(0, i)$ and $\mathbf{N}(-n+1, n-1+k-i)$, respectively.

Theorem 8 We have

$$
\sum_{n \geq 0} \sum_{k \geq 0} N(-n, n+k) x^{n} y^{k}=\frac{\mathcal{P}(y)}{1-\mathcal{P}(y) x} .
$$

Proof The recurrence relation (24) implies $\sum_{k \geq 0} N(-n, n+k) y^{k}=\mathcal{P}(y)^{n+1}$ which gives the two-variable generating function.

Combining (6) with (25a) we obtain

$$
\sum_{n, k \geq 0} N(-n, n+k) x^{n} y^{k}=\frac{2}{1-y+\sqrt{1-6 y-3 y^{2}}-2 x} .
$$

By Theorem 8 and Theorem 1, we have for $n, k \geq 0$,

$$
N(-n, n+k)=\frac{n+1}{k+n+1} \sum_{s=0}^{k} \sum_{j=0}^{s}\left(\begin{array}{c}
k+n+1 \\
j
\end{array}\right)\left(\begin{array}{c}
j \\
s-j
\end{array}\right)\left(\begin{array}{c}
2 k+n-s \\
k+n
\end{array}\right) \text {. }
$$


5.3 The Lattice $\mathcal{L}^{*}$

Theorem 9 The following statements are true and equivalent.

(a) (recurrence relation) For $n \geq 0$ we have $U(n, n)=U(n)$, and for $k \geq 1$,

$$
U(n+k, n)=\sum_{i=0}^{n} U(i, i) U(n+k-i-1, n-i) .
$$

(b) (generating function)

$$
\mathcal{U}(x, y)=\sum_{k \geq 0} \sum_{n \geq 0} U(n+k, n) x^{n} y^{k}=\frac{\mathcal{U}(x)}{1-\mathcal{U}(x) y} .
$$

(c) (exact formula)

$$
U(n+k, n)=\sum_{p=0}^{k+1} \sum_{j=0}^{n-p} \sum_{i=0}^{n-p-j} \frac{p}{n}\left(\begin{array}{c}
k+1 \\
p
\end{array}\right)\left(\begin{array}{l}
n \\
j
\end{array}\right)\left(\begin{array}{c}
n \\
i
\end{array}\right)\left(\begin{array}{c}
i \\
n-p-j-i
\end{array}\right) 3^{i+p+j}
$$

Proof (a) Observe that every path $\pi$ from $\mathbf{U}(n+k, n)$ must have a horizontal step $h_{i}=$ ( $(i, i),(i+1, i))$, where $i=\max \left\{j: h_{j} \in \pi\right\}$. Thus $\pi$ can be uniquely decomposed as $\pi_{1} h_{i} \pi_{2}$, where $\pi_{1}$ and $\pi_{2}$ are paths from $\mathbf{U}(i, i)$ and $\mathbf{U}(n+k-i-1, n-i)$, respectively. (b) The recurrence relation implies $\sum_{n \geq 0} U(n+k, n) x^{n}=\mathcal{U}(x)^{k+1}$. (c) Combining Theorem 4 with (27) we obtain the exact formula.

\subsection{The Lattice $\mathcal{L}$}

Here we give the array $(S(i, j))_{i, j}$ for $0 \leq i \leq 4$ and $0 \leq j \leq 6$,

$$
\left(\begin{array}{lllllll}
1 & 3 & 15 & 81 & 459 & 2673 & 15849 \\
6 & 33 & 189 & 1107 & 6588 & 39663 & 240894 \\
60 & 378 & 2349 & 14553 & 90207 & 560115 & 3485187 \\
675 & 4509 & 29403 & 189351 & 1211031 & 7715331 & 49045662 \\
7992 & 55188 & 371358 & 2458998 & 16112925 & 104838219 & 678790125
\end{array}\right) .
$$

Proposition $7 S(0,0)=1$, and for $n, k \geq 0$ such that $(n, k) \neq(0,0)$, we have

$$
\begin{aligned}
S(n, k) & =\sum_{i=0}^{k} S(0, i) P(n-1, k-i)+\sum_{i=0}^{k-1} S(0, i) P(n-1, k-1-i), \\
S(-n, n+k) & =\sum_{i=0}^{k} S(0, i) N(-n+1, n+k-i-1), \\
S(n+k, n) & =\sum_{i=0}^{n} S(i, i) U(n+k-i-1, n-i) .
\end{aligned}
$$


Proof The case $n=0$ is trivial. Let $n \geq 1$. The case (a) may be handled in much the same way as the case (a) of Proposition 5 and the case (b) as the Proposition 6. We only note that in (b) the number of paths from the ending point of a sloping step $s_{i}=((0, i),(-1, i+1))$ to $(-n, n+k)$ is $N(-n+1, n+k-i-1)$ which can be easily shown by rotating any such path by 180 degrees about the point $(-n, n+k)$. The recurrence (c) can be handled in much the same way as (a) of Theorem 9.

Theorem 10 We have

$$
\begin{aligned}
& \mathcal{S}_{1}(x, y)=\sum_{n \geq 0} \sum_{k \geq 0} S(n, k) y^{k} x^{n}=\frac{\mathcal{S}(y)}{1-x \mathcal{P}(y)(1+y)}, \\
& \mathcal{S}_{2}(x, y)=\sum_{n \geq 0} \sum_{k \geq 0} S(-n, n+k) y^{k} x^{n}=\frac{\mathcal{S}(y)}{1-x \mathcal{P}(y)} \\
& \mathcal{S}_{3}(x, y)=\sum_{n \geq 0} \sum_{k \geq 0} S(n+k, n) y^{k} x^{n}=\frac{\widehat{\mathcal{S}}(x)}{1-y \mathcal{U}(x)} .
\end{aligned}
$$

Proof The recurrence relations (28a), (28b) and (28c) imply

$$
\begin{aligned}
\sum_{k \geq 0} S(n, k) y^{k} & =\mathcal{S}(y) \mathcal{P}(y)^{n}(1+y)^{n}, \\
\sum_{k \geq 0} S(-n, n+k) y^{k} & =\mathcal{S}(y) \mathcal{P}(y)^{n}, \\
\sum_{n \geq 0} S(n+k, n) x^{n} & =\widehat{\mathcal{S}}(x) \mathcal{U}(x)^{k} .
\end{aligned}
$$

Simplifying (29a) and (29b) we obtain

$$
\begin{aligned}
& \mathcal{S}_{1}(x, y)=\frac{2 y}{\sqrt{1-6 y-3 y^{2}}\left(2 y+x\left(-1+y+\sqrt{1-6 y-3 y^{2}}\right)\right)} \\
& \mathcal{S}_{2}(x, y)=\frac{2 y(1+y)}{\sqrt{1-6 y-3 y^{2}}\left(2 y(1+y)+x\left(-1+y+\sqrt{1-6 y-3 y^{2}}\right)\right)}
\end{aligned}
$$

\subsection{The Lattice $\mathcal{L}_{n}$}

For all $n, k \geq 0$ and $0 \leq p \leq n$, let us denote by $\mathbf{R}(n, k, p)$ the family of paths running from $(0,0)$ to $(p, k)$ which lie in the lattice $\mathcal{L}_{n}=\{(i, j): 0 \leq i \leq n, j \geq 0\}$. We denote by $R(n, k, p)$ the size of $\mathbf{R}(n, k, p)$. An example of a path from $\mathbf{R}(4,3,3)$ is given in Fig. 6.

Theorem 11 The following statements are true and equivalent. 
Fig. 6 A path from $\mathbf{R}(4,3,3)$

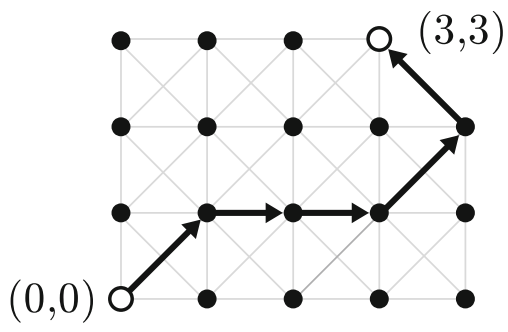

(a) (recurrence relation) For $n, k \geq 0$ we have $R(n, k, 0)=T_{n}(k)$, and for $1 \leq$ $p \leq n$ we have

$$
\begin{aligned}
R(n, k, p)= & \sum_{i=0}^{k} T_{n}(i) R(n-1, k-i, p-1) \\
& +\sum_{i=0}^{k-1} T_{n}(i) R(n-1, k-1-i, p-1) .
\end{aligned}
$$

(b) (generating function) For $n \geq 0$ and $0 \leq p \leq n$, we have

$$
\sum_{k \geq 0} R(n, k, p) z^{k}=(1+z)^{p} \prod_{i=0}^{p} \mathcal{T}_{n-i}(z)
$$

Proof To show the recurrence relation we use the same argument as in (a) of the proof of Proposition 5. Let $\mathcal{R}_{n, p}(z)$ denote the left-hand side of (32). The recurrence relation (31) implies the functional equation $\mathcal{R}_{n, p}(z)=(1+z) \mathcal{T}_{n}(z) \mathcal{R}_{n-1, p-1}(z)$ with $\mathcal{R}_{n-p, 0}(z)=\mathcal{T}_{n-p}(z)$.

\section{Paths in a Rectangle}

In this section we derive the generating function for the numbers $R(n, k)$ of paths from the origin to $(n, k)$ which lie entirely in the lattice $\mathcal{L}_{n}=\left\{(i, j) \in \mathbb{N}^{2}: 0 \leq i \leq n\right\}$. We denote by $\mathbf{R}(n, k)$ the family of these paths. Let

$$
\mathcal{R}_{n}(z)=\sum_{k \geq 0} R(n, k) z^{k}
$$

\subsection{The First Approach}

Theorem 12 For $n \geq 0$ we have

$$
\mathcal{R}_{n}(z)=\frac{2^{n+2}(1+z)^{n} \sqrt{1-6 z-3 z^{2}}}{\left(1-z+\sqrt{1-6 z-3 z^{2}}\right)^{n+2}-\left(1-z-\sqrt{1-6 z-3 z^{2}}\right)^{n+2}} .
$$


Proof Let us consider the numbers $R(n, k, p)$ from Sect. 5.5. Observe that $R(n, k)=$ $R(n, k, n)$. Therefore, applying Theorem 11 we obtain

$$
\sum_{k \geq 0} R(n, k) z^{k}=(1+z)^{n} \prod_{i=0}^{n} \mathcal{T}_{i}(z),
$$

where $\mathcal{T}_{i}(z)$ is given in Theorem 2.

Recall, by the reciprocal of a formal power series $\mathcal{F}(x)=\sum_{n \geq 0} F(n) x^{n}$ such that $F(0) \neq 0$, we mean the formal power series $1 / \mathcal{F}(x)$ such that $\mathcal{F}(x) \cdot(1 / \mathcal{F}(x))=1$.

Corollary 6 We have

$$
\sum_{n \geq 0} \frac{y^{n}}{\mathcal{R}_{n}(z)}=\frac{(1+z)(1-z-y z)}{1-y-y z+z+y^{2} z} .
$$

Proof By (33) we see that

$$
\frac{1}{\mathcal{R}_{n}(z)}=\frac{(1+z)^{2}}{\sqrt{1-6 z-3 z^{2}}}\left(\rho_{+}^{n+2}-\rho_{-}^{n+2}\right),
$$

where $\rho_{ \pm}=\left(1-z \pm \sqrt{1-6 z-3 z^{2}}\right) /(2(1+z))$. Multiplying both sides by $y^{n}$ and summing over $n \geq 0$ we obtain the formula.

The function (35) codes $R(n, k)$ for any $n, k \geq 0$, in the sense that

$$
\sum_{n \geq 0} \frac{y^{n}}{\sum_{k \geq 0} R(n, k) z^{k}}=\frac{(1+z)(1-z-y z)}{1-y-y z+z+y^{2} z} .
$$

Problem 3 There are still several questions about the numbers $R(n, k)$. What is a generating function for the numbers $R(n, n)$ ? Is there are simple exact formula for $R(n, k)$ ? What is the asymptotic behaviour of $R(n, k)$ ?

Corollary 7 For all $n, k \geq 0$ we have

$$
\begin{gathered}
{\left[z^{k}\right] \frac{1}{\mathcal{R}_{n}(z)}=(-1)^{k}(S(n+1-k, k)-2 S(n+2-k, k-1)} \\
+S(n+3-k, k-2)) .
\end{gathered}
$$

Proof By (3) we obtain $1 / \mathcal{R}_{n}(z)=(1+z)^{2} \mathcal{S}_{\langle n+1\rangle}(-z)$. Comparing the coefficient of $z^{k}$ on both sides we get the formula.

Proposition $8 R(n, 0)=R(0, n)=1$, and for all $n, k \geq 1$ we have

$$
R(n, k)=\sum_{i=0}^{k-1}(-1)^{i} r(i) R(n, k-i-1),
$$


where

$$
r(i)=(S(n-i, i+1)-2 S(n+1-i, i)+S(n+2-i, i-1)) .
$$

Proof Let $r(n, k)=\left[z^{k}\right]\left(1 / \mathcal{R}_{n}(z)\right)$. Using the recurrence relation for a reciprocal of a formal power series we obtain

$$
R(n, k)=\frac{-1}{r(n, 0)} \sum_{i \geq 1} r(n, i) R(n, k-i)
$$

Applying Corollary 7 the formula follows.

Problem 4 Find a combinatorial proof of (37).

\subsection{The Second Approach}

For all $n, k \geq 0$, let $K(n, k)$ denote the number of paths from $(n+1,0)$ to $(n, k)$ that lie in the positive lattice $\mathcal{L}^{+}$. One can show that for $k \geq 1$ we have

$$
K(n, k)=S(-1, k)-\sum_{j=0}^{k-n-2} N(-n-1, n+1+j) S(n+1, k-n-2-j)
$$

It follows that $K(n, k)=S(-1, k)$ for $k<n+2$. By Theorem 8 and Theorem 10, we derive

$$
\mathcal{K}_{n}(z)=\sum_{k \geq 0} K(n, k) z^{k}=z \mathcal{S}(z) \mathcal{P}(z)\left(1-z^{n+1}(1+z)^{n+1} \mathcal{P}(z)^{2 n+2}\right)
$$

Proposition 9 For all $n, k \geq 0$ we have $R(n, 0)=R(0, k)=1$, and

$$
R(n, k)=P(n, k)-\sum_{i=0}^{k-1} R(n, i) K(n, k-i)-\sum_{i=0}^{k-2} R(n, i) K(n, k-i-1) .
$$

Proof Observe that $\mathbf{R}(n, k)$ is a subset of the set $\mathbf{P}(n, k)$. To get the size of $\mathbf{R}(n, k)$ we need to remove from $\mathbf{P}(n, k)$ these paths, denoted as the set $A$, which contain lattice points $\{(i, j): i>n, j \geq 0\}$. We partition $A$ into $k$ sets $H_{0}, H_{1}, \ldots, H_{k-1}$ and $(k-1)$ sets $D_{0}, D_{1}, \ldots, D_{k-2}$ as follows. The set $H_{i}$ contains these paths $\pi$ from $\mathbf{P}(n, k)$ such that $\pi$ consists of horizontal step $((n, i),(n+1, i))$ and $D_{i}$ these $\pi \in \mathbf{P}(n, k)$ such that $\pi$ consists of diagonal step $((n, i),(n+1, i+1))$. The sizes of $H_{i}$ and $D_{i}$ are $R(n, i) K(n, k-i)$ and $R(n, i) K(n, k-i-1)$, due to the definition of the numbers $K$. 
Theorem 13 For $n \geq 0$ we have

$$
\mathcal{R}_{n}(z)=\frac{(1+z)^{n} \mathcal{P}(z)^{n+1}}{1+z(1+z) \mathcal{S}(z) \mathcal{P}(z)\left(1-z^{n+1}(1+z)^{n+1} \mathcal{P}(z)^{2 n+2}\right)}
$$

Proof The recurrence relation (39) implies the functional equation $\mathcal{R}_{n}(z)=\mathcal{P}_{n}(z)-$ $\mathcal{R}_{n}(z) \mathcal{K}_{n}(z)-z \mathcal{R}_{n}(z) \mathcal{K}_{n}(z)$ which implies

$$
\mathcal{R}_{n}(z)=\frac{\mathcal{P}_{n}(z)}{1+\mathcal{K}_{n}(z)(1+z)}
$$

Simplifying and substituting (23b) and (38) to the above we obtain the formula.

\subsection{The Third Approach}

Proposition 10 For all $n, k \geq 0$ we have $R(n, 0)=R(0, k)=1$, and

$$
\begin{aligned}
R(n, k)= & S(n, k)-\sum_{i=0}^{k-1} R(n, i) S(-1, k-i) \\
& -\sum_{i=0}^{k-2} R(n, i) S(-1, k-1-i)-\sum_{i=0}^{k-1} T_{n}(i) S(n+1, k-1-i) .
\end{aligned}
$$

Proof The idea of the proof is similar to that one of Proposition 9. In this case we need to show that $\mathbf{R}(n, k)$ is a subfamily of $\mathbf{S}(n, k)$ without these paths: (a) $\pi \in \mathbf{S}(n, k)$ such that $\pi$ contains a horizontal step $((n, i),(n+1, i))$ for some $i=0,1, \ldots, k-1$, or a diagonal step $((n, i),(n+1, i+1))$ for some $i=0,1, \ldots, k-2$, and the previous step of $\pi$ lie entirely in $\mathcal{L}_{n}$, and (b) these paths $v$ from $\mathcal{S}(n, k)$ such that $v$ contains a sloping step $((0, i),(-1, i+1))$ for some $i=0,1, \ldots, k-1$, and the previous steps of $\pi$ lie entirely in $\mathcal{L}_{n}$.

Theorem 14 For $n \geq 0$ we have

$$
\mathcal{R}_{n}(z)=\frac{\mathcal{S}(z) \mathcal{P}(z)^{n}(1+z)^{n}\left(1-z \mathcal{T}_{n}(z) \mathcal{P}(z)(1+z)\right)}{1+z(1+z) \mathcal{S}(z) \mathcal{P}(z)} .
$$

Proof The recurrence relation (41) implies that $\mathcal{R}_{n}(z)$ is equal to

$$
\sum_{k \geq 0} S(n, k) z^{k}-z(z+1) \mathcal{R}_{n}(z) \sum_{k \geq 0} S(-1, k+1) z^{k}-z \mathcal{T}_{n}(z) \sum_{k \geq 0} S(n+1, k) z^{k}
$$

Simplifying and substituting (30a) the formula follows. 


\section{Counting Paths by Length}

In previous sections we consider the numbers of path from the origin to specified lattice point. Another approach is to count lattice paths by length. Namely, let $\mathbf{G}(k)$ denote the family of lattice paths of length $k$ running from the origin to any point of the $y$-axis and whose points lie in the positive lattice $\mathcal{L}^{+}$. We write $G(k)$ for the size of $\mathbf{G}(k)$.

Theorem 15 The following statements are true and equivalent.

(a) (recurrence relation) $G(0)=1$, and for $k \geq 0$ we have

$$
G(k)=G(k-1)+2 \sum_{i=0}^{k-2} G(i) G(k-2-i) .
$$

(b) (generating function)

$$
\mathcal{G}(z)=\sum_{k \geq 0} G(k) z^{k}=\frac{1-t-\sqrt{1-2 z-7 z^{2}}}{4 z^{2}} .
$$

(c) (functional equation)

$$
\mathcal{G}(t)=1+z \mathcal{G}(t)+2 z^{2} \mathcal{G}(z)^{2} .
$$

(d) (exact formula)

$$
\left[z^{n}\right] \mathcal{G}(z)^{d}=\frac{d}{n+d} \sum_{j=0}^{n}\left(\begin{array}{c}
n+d \\
j
\end{array}\right)\left(\begin{array}{c}
j \\
n-j
\end{array}\right) 2^{n-j} .
$$

Proof For $k=0,1$ it is true, thus take $k \geq 2$. To show the recurrence relation, observe that the last step of any path $\pi$ from $\mathbf{G}(k)$ is either vertical or sloping. In the firs case, the number of paths from $\mathbf{G}(k)$ whose the last step is vertical is $G(k-1)$. In the second case, observe that $\pi$ must have a horizontal step $h_{j}=((0, j),(1, j))$ or a diagonal $d_{j}=((0, j),(1, j+1))$, where $j$ is the maximal such number. It implies that $\pi$ has unique decomposition into $\pi_{1} s \pi_{2}$, where $s \in\left\{h_{j}, d_{j}\right\}$ and $\pi_{1}, \pi_{2}$ are paths of length $i$ and $k-2-i$, respectively, for certain $i \in\{0,1, \ldots, k-2\}$. Thus the number of paths from $\mathbf{G}(k)$ whose the last step is a sloping one is $2 G(i) G(k-2-i)$.

From the recurrence we obtain the functional equation which implies the generating function. The exact formula is inferred from the Lagrange Inversion Formula applied to the functional equation with the substitution $u(z)=z \mathcal{G}(z)$ and $\phi(u)=1+u+2 u^{2}$ (see the proof of Theorem 1 for more details).

The function $\mathcal{G}(z)$ is a continued fraction

$$
\mathcal{G}(z)=\frac{1}{1-z-\frac{2 z^{2}}{1-z-\frac{2 z^{2}}{1-z-\frac{2 z^{2}}{1-z-\ldots}}}}
$$


The Hankel transform [7,17] of a given sequence $\left(f_{0}, f_{1}, \ldots\right)$ is the sequence of Hankel determinants $\left(h_{n}\right)_{n \geq 0}$, where $h_{n}=\left|f_{i+j}\right|_{0 \leq i, j \leq n}$. Due to the form of (43) the Hankel transform of $(G(n))_{n \geq 0}$ is

$$
h_{n}=\prod_{i=1}^{n} 2^{n-i+1}=2^{\left(\begin{array}{c}
n+1 \\
2
\end{array}\right)} .
$$

The denominator polynomials $D_{n}(z)$ are given by $D_{n}(z)=(1-z) D_{n-1}-2 z^{2} D_{n-2}$ with $D_{0}(z)=1$ and $D_{1}(z)=1-z-2 z^{2}$.

Remark The sequence

$$
(G(k))_{k \geq 0}=(1,1,3,7,21,61,191,603,1961,6457,21595,72975, \ldots)
$$

is denoted by A025235 in OEIS [13].

Let $\mathbf{G}(n, k)$ denote the family of lattice paths of length $k$ from the origin to any point of the line $x=n$ and whose points lie in the positive lattice $\mathcal{L}^{+}$. We write $G(n, k)$ for the size of $\mathbf{G}(n, k)$. Observe that every path from $G(n, k)$, where $n>0$ must have a horizontal or a diagonal step that begins on the line $y=0$ and ends on $y=1$. Thus the numbers $G(n, k)$ satisfy the following recurrence relation

$$
G(n, k)=2 \sum_{i=0}^{k} G(0, i) G(n-1, k-1-i) .
$$

From the recurrence relation we obtain

$$
\begin{aligned}
\sum_{k \geq 0} G(n, k) z^{k} & =2^{n} z^{n} \mathcal{G}(z)^{n+1}, \\
\sum_{n \geq 0} \sum_{k \geq 0} G(n, k) z^{k} t^{n} & =\frac{\mathcal{G}(z)}{1-z 2 \mathcal{G}(z) t} .
\end{aligned}
$$

\section{Riordan Arrays}

The Riordan group $[12,14,15]$ is a set of infinite lower-triangular matrices. Let $g(z)=$ $\sum_{n \geq 0} g_{n} z^{n}$ with $g_{0} \neq 0$ and $f(z)=\sum_{n \geq 1} f_{n} z^{n}$ with $f_{1} \neq 0$. We associate with $g(z)$ and $f(z)$ the matrix, denoted by $(g, f)$, whose $(i, j)$ th element is $\left[z^{i}\right] g(z) f(z)^{j}$ for $i, j \geq 0$. The matrix $(g, f)$ is called a proper Riordan array.

These arrays have been intensively studied in the literature. It turns out that most of the numbers studied in this paper have a representation as a Riordan array. For instance, we have

$$
(S(i-2 j, j))_{i, j \geq 0}=\left(\frac{1}{1-z}, z \frac{1+z+z^{2}}{1-z}\right)
$$


which follows from (2). Indeed,

$$
S(i-2 j, j)=\left[z^{i}\right] \frac{1}{1-z}\left(z \frac{1+z+z^{2}}{1-z}\right)^{j}=\left[z^{i-j}\right] \frac{\left(1+z+z^{2}\right)^{j}}{(1-z)^{j+1}} .
$$

The following Riordan arrays follow from the generating functions inferred in previous sections,

$$
\begin{aligned}
(S(j, i-j))_{i, j \geq 0} & =(\mathcal{S}(z), z(1+z) \mathcal{P}(z)), \\
(S(-j, i))_{i, j \geq 0} & =(\mathcal{S}(z), z \mathcal{P}(z)), \\
(S(i, i-j))_{i, j \geq 0} & =(\widehat{\mathcal{S}}(z), z \mathcal{U}(z)), \\
(P(j, i-j))_{i, j \geq 0} & =(\mathcal{P}(z), z(1+z) \mathcal{P}(z)), \\
(N(-j, i))_{i, j \geq 0} & =(\mathcal{P}(z), z \mathcal{P}(z)), \\
(U(i, i-j))_{i, j \geq 0} & =(\mathcal{U}(z), z \mathcal{U}(z)), \\
(G(j, i))_{i, j \geq 0} & =(\mathcal{G}(z), z 2 \mathcal{G}(z)) .
\end{aligned}
$$

The inverse Riordan array to $(g, f)$, denoted by $(g, h)^{-1}$, is $(1 /(g \circ \bar{f}, \bar{f})$, where $\bar{f}$ is the compositional inverse of $f$, i.e., $f(\bar{f}(z))=\bar{f}(f(z))=z$. For instance, we have

$$
\begin{aligned}
(N(-j, i))_{i, j \geq 0}^{-1} & =\left(\frac{1-z}{1+z+z^{2}}, \frac{z(1-z)}{1+z+z^{2}}\right), \\
(G(j, i))_{i, j \geq 0}^{-1} & =\left(\frac{1}{1+z+z^{2}}, \frac{z}{1+z+z^{2}}\right) .
\end{aligned}
$$

Due to the correspondences above, we may expect several new questions which appear in the context of the Riordan arrays, continued fractions, Hankel transforms etc. and we left it for further investigation.

Acknowledgments I would like to thank anonymous referees for very careful reading of the manuscript and helpful suggestions that improved the presentation of the paper.

Open Access This article is distributed under the terms of the Creative Commons Attribution License which permits any use, distribution, and reproduction in any medium, provided the original author(s) and the source are credited.

\section{References}

1. Banderier, C., Schwer, S.: Why Delannoy numbers. J. Stat. Plan. Inference 135(1), 40-54 (2005)

2. Chen, W.Y.C., Li, N.Y., Shapiro, L.W., Yan, S.H.F.: Matrix identities on weighted partial Motzkin paths. Eur. J. Comb. 28, 1196-1207 (2007)

3. Comtet, L.: Advanced Combinatorics: The Art of Finite and Infinite Expansions. D. Reidel Publishing Company, Boston (1974)

4. Drake, B.: Limits of areas under lattice paths. Discret. Math. 309(12), 3936-3953 (2009) 
5. Dziemiańczuk, M.: A bijection between weighted free (3,3)-Motzking paths and generalized Delannoy paths with four types of steps (2012). Preprint, submitted for publication

6. Flajolet, P., Sedgewick, R.: Analytic Combinatorics. Cambridge University Press, Cambridge (2009)

7. Krattenthaler, C.: Advanced determinant calculus: a complement. Linear Algebra Appl. 411, 68-166 (2005)

8. Kruchinin, V.: Compositae and their properties Preprint arXiv:1103.2582 (14 Mar 2011)

9. Lehner, F.: Cumulants, lattice paths, and orthogonal polynomials. Discret. Math. 270(1-3), 177-191 (2003)

10. Merlini, D., Sprugnoli, R., Verri, M.C.: Lagrange inversion: when and how. Acta Appl. Math. 94, 233-249 (2006)

11. Merlini, D., Sprugnoli, R., Verri, M.C.: The method of coefficients. Am. Math. Mon. 114(1), 24-57 (2007)

12. Shapiro, L.W., Getu, S., Woan, W.J., Woodson, L.: The Riordan group. Discret. Appl. Math. 34, 229-239 (1991)

13. Sloane, N.J.A.: The on-line encyclopedia of integer sequences. Published electronically at http://oeis. org/

14. Sprugnoli, R.: Riordan arrays and combinatorial sums. Discret. Math. 32, 267-290 (1994)

15. Sprugnoli, R.: A bibliography on Riordan arrays. Published electronically at http://www.dsi.unifi.it/ $\sim$ resp/BibRioMio.pdf. (2008)

16. Stanley, R.P.: Enumerative Combinatorics, vol. II. Cambridge University Press, Cambridge (1999)

17. Wall, H.S.: Analytic Theory of Continued Fractions. AMS Chelsea Publishing, New York (2000)

18. Wilf, H.S.: Generatingfunctionology. Academic Press, Boston (1994) 